\title{
HACIA UNA EDUCACIÓN RELIGIOSA GENERADORA DE CONVIVIALIDAD Y RESPETO EN LAS DIVERSIDADES
}

\author{
FOR A RELIGIOUS EDUCATION GENERATOR OF CONVIVIALITY AND \\ RESPECT IN DIVERSITIES
}

POR UM ENSINO RELIGIOSO GERADOR DE CONVIVIALIDADE E RESPEITO NAS DIVERSIDADES

RESUMEN: El presente trabajo tiene como objetivo reconocer y subrayar la importancia de los procesos educativos para la formación de la solidaridad y el respeto en la convivialidad, aspectos imprescindibles para el diálogo interreligioso, tema central de la reflexión. A través de la observación de la realidad costarricense y de un análisis bibliográfico, la autora resalta la importancia que adquieren los procesos educativos - en los diferentes espacios de la cotidianidad en donde se da la enseñanza y el aprendizaje -, para el ejercício del diálogo y la convivencia respetuosa. Este reto se presenta de manera especial en Costa Rica, donde la religión católica sigue siendola religión oficial del Estadoy los planes curriculares no contemplan satisfactoriamente las exigencias de un escenario pluricultural y multirreligioso. El desarrollo de una epistemología indócil y la implementación de metodologías relacionales son los principales desafíos para la educación religiosa en la actualidad.

Palabras claves: educación religiosa; diversidades; interculturalidad; multirreligiosidad; convivialidad.
ABSTRACT: The present work aims to recognize and emphasize the importance of educational processes for the formation of solidarity and respect in conviviality, indispensable aspects for interreligious dialogue, a central theme of reflection. Through the observation of Costa Rican reality and a bibliographical analysis, the author emphasizes the importance of the educational processes - in the different spaces of everyday life in which teaching and learning happens -, for the exercise of dialogue and respectful coexistence. This challenge is particularly evident in Costa Rica, where the Catholic

religion continues to be the official religion of the State and curricula do not satisfactorily meet the requirements of a multicultural and multireligious scenario. The development of an indocletizing epistemology and the implementation of relational methodologies are the main challenges for religious education today.

Keywords: religious education; diversities; interculturality; multireligiosity; conviviality.

RESUMO: O presente trabalho tem como objetivo reconhecer e ressaltar a importância dos processos educativos para a formação da solidariedade e o respeito na convivialidade, aspectos imprescindíveis para o diálogo inter-religioso, tema central da reflexão. Através da observação da realidade costarriquenha e de uma análise bibliográfica, a autora enfatiza a importância que adquirem os processos educativos - nos diferentes espaços da cotidianidade nos quais acontece o ensino e a aprendizagem -, para o exercício do diálogo e da convivência respeitosa. Este desafio se apresenta de maneira especial na Costa Rica, onde a religião católica continua sendo a religião oficial do Estado e os planos curriculares não contemplam satisfatoriamente as exigências de um cenário pluricultural e multirreligioso. $\mathrm{O}$ desenvolvimento de uma epistemologia indócil e o implemento de metodologias relacionais são os principais desafios para a educação religiosa na atualidade.

Palabras chaves: educação religiosa; diversidades; interculturalidade; multirreligiosidade; convivialidade. 


\section{INTRODUCCIÓN}

La presente reflexión nace de una preocupación que viene acompañando las personas educadoras y estudiosas de los asuntos religiosos en Costa Rica: la educación religiosa en los centros educativos públicos. Cada vez más es posible comprobar a través de los censos, las encuestas y en la maravillosa experiencia cotidiana de convivio intercultural, que Costa Rica es un país no solamente portador de una belleza natural sorprendente, sino también es una nación multiétnica, multicultural y con una diversidad religiosa incontestable. Sin embargo, reconocer la belleza natural y la riqueza humana que caracteriza la diversidad costarricense, no significa que la coexistencia de las diferentes etnias, culturas y religiones se traduzca en una convivencia armoniosa y respetuosa en el país de forma consistente.

La generación y la nutrición de la solidaridad, el respeto y la convivencia pasan por los procesos educativos, es decir, las personas aprenden a ser respetuosas y solidarias en la convivencia y, además, estos son aprendizajes que deben iniciarse en la más temprana edad y prolongarse durante toda la vida. Raúl Fornet-Betancourt, al referirse a la convivencia, señala que esta no es una condición que el ser humano pueda elegir, es decir, la vida humana implica necesariamente "estar y actuar en un ámbito común, en un mundo en el que desde siempre ya estamos con los demás". (FORNET-BETANCOURT: 2011, p. 21). Por este motivo, la convivencia implica diferentes formas de relacionamiento humano y comportamientos diversos y antagónicos, como lo son el amor y el odio, la confianza y la desconfianza, la cooperación y la rivalidad, por ejemplo (FORNETBETANCOURT: 2011, p. 22).

En una entrevista sobre este tema, el autor no solamente explica lo que entiende por convivencia, sino también sugiere el vocablo convivialidad para expresar una convivencia basada en la justicia, la solidaridad y el reconocimiento respetuoso de las diversidades:

La vida humana es convivencia. Nadie puede vivir su vida sin convivir; pero, por eso mismo, es la convivencia también el lugar que puede acabar con o reducir a un mínimo la vida de quienes consideramos otros. Consciente de esto, la filosofía intercultural propone que el desafío radica precisamente en convertir la convivencia fáctica, el factum, de la convivencia, en una experiencia de convivialidad, entendiendo por esta última ese proceso lento de ir logrando un ambiente de paz y amistad en nuestras relaciones sociales, políticas o culturales. (BERISSO; GIULIANO: 2015, p. 157)

El concepto convivialidad, propuesto por primera vez por Iván Illich, significa la calidad y la calidez de la 
convivencia, es decir, la habilidad humana para con-vivir de forma sana. Desde una perspectiva educativa, se puede afirmar que aprender la convivialidad tiene que ver con aprender el lenguaje del convivir, que debe acompañar el aprendizaje del lenguaje humano. Humberto Maturana, al concebir la educación como mediación para formar destrezas emocionales y como proceso de transformación en la convivencia, condiciona el con-vivir al ejercicio de la conversación:

Los niños crecen como seres humanos entrelazando lenguaje y emocionalismo en su vida, en un flujo continuo de trenzamiento de dominios relacionales (emociones) y recurrentes coordinaciones consensuales de conductas (lenguaje) que denominamos conversaciones. Todo lo que nosotros, los seres humanos, hacemos como tales, lo hacemos en conversaciones. $\mathrm{Y}$ aquello que no hacemos en conversaciones, de hecho no lo hacemos como seres humanos. (...) Puesto que todo lo que hacemos como seres humanos lo hacemos en conversaciones, $\mathrm{y}$ las conversaciones se producen en el fluir de nuestras interacciones, todo lo que hacemos en conversaciones modula el flujo de cambios estructurales y llegamos a ser en nuestro flujo estructural según sean las conversaciones en las cuales participamos. En el fluir de nuestra vida no hay conversaciones triviales. (MATURANA: 2004, p. 4)

De forma similar, Paulo Freire concibe el diálogo, o la conversación, como un acto de amor que facilita el crecimiento personal, comunitario y social y posibilita asumir los problemas colectivos para buscar posibles consensos. Desde esta perspectiva, las crisis y los conflictos no son vistos desde lo negativo, sino como posibilidad de encuentro y transformación positiva: "el diálogo es el encuentro amoroso de las personas que, mediatizadas por el mundo, lo pronuncian, es decir, lo transforman y, transformándolo, lo humanizan hacia la humanización de todos. Ese encuentro amoroso no puede ser, por eso, un encuentro de inconciliables". (FREIRE: 1983, p. 43)

Con esto, se resalta la importancia de los procesos educativos - en los diferentes espacios de la cotidianidad en los cuales se aprende y se enseña -, para el ejercicio de la conversación y, consecuentemente, para la formación de la solidaridad y el respeto en la convivialidad, aspectos imprescindibles para el diálogo interreligioso, tema central de esta reflexión. Además, es necesario destacar la importancia que adquiere la educación religiosa para la formación (o no) de estas posturas, reto que se presenta de manera especial en Costa Rica, donde la religión católica sigue siendo la religión oficial del Estado y los planes curriculares de educación religiosa todavía no han incorporado los cambios establecidos por la resolución 
$1 \mathrm{Al}$ inicio del 2010, a través de la Resolución 2023, la Sala IV anuló un artículo de la ley de Carrera Docente que permitía a los obispos católicos autorizar o vetar el nombramiento de profesores de religión pagados por el MEP. El artículo declarado inconstitucional es el 34 bis, que establecía como requisito indispensable la autorización previa de la Conferencia Episcopal para seleccionar el personal dedicado a la educación religiosa en escuelas y colegios de carácter público. de la Sala Constitucional ${ }^{1}$ que deroga la Misio Canónica. Es evidente la carencia de una adecuación curricular coherente con el escenario pluricultural y multirreligioso costarricense.

En este contexto, optamos por problematizar la expresión educación inclusiva tan frecuente en los discursos educativos actuales. Esto porque hablar de inclusión educativa significa necesariamente imaginarse una frontera, espacial o simbólica, desde la cual se incluye o se excluye a las personas involucradas en el proceso de aprendencia y enseñanza. De esta forma, si hay una frontera educativa, necesariamente existe un grupo privilegiado que tiene la potestad para incluir (o no) a otros grupos considerados minoritarios y/o diferentes del centro de referencia; es decir, la inclusión en principio no eliminaría el ejercicio abusivo del poder en la educación. Además, hay que preguntarse quién o quiénes incluyen, en qué espacio o en qué sistema se incluye, y para qué se incluye.

Por otro lado, si se aprende realmente a con-vivir, a vivir-con y si se aprende a celebrar las diversidades, ya no será necesario hablar de inclusión educativa, ya que todos y todas serán partícipes y la inclusión será una realidad construida y celebrada pedagógicamente. La clave es, pues, la promoción de cambios relacionales más profundos en la educación para generar convivialidades - convivencias armoniosas -, entendiendo que los conflictos y las diversidades son ingredientes necesarios de la armonía. Por esto no asumimos la expresión educación inclusiva, sino que la reflexión acontece desde una perspectiva pedagógica nutriente de convivialidad y respeto en las diversidades. El respeto en las diversidades presupone que la propia educación se ubica y se desenvuelve dentro de escenarios en donde las diversidades se constituyen una evidencia y una riqueza relacional.

El contexto multirreligioso que caracteriza Costa Rica ha sido no solamente un desafío para la vivencia y convivencia entre las diversas creencias y no-creencias, sino también una preocupación desde los espacios académicos en el sentido de visibilizar y analizar el estado de lo religioso en el país. Laura Fuentes, investigadora coordinadora de una encuesta sobre la situación religiosa en Costa Rica, realizada por la Escuela Ecuménica de Ciencias de la Religión, apunta la existencia de un menú de creencias decurrentes de los cambios en el escenario religioso costarricense:

La mayoría de los creyentes entrevistados tiende más a elegir qué creer, cómo hacerlo, y cómo practicarlo que a seguir al pie de la letra lo establecido por las instituciones religiosas, de esta forma, las creencias tradicionales pueden ceder el paso a interpretaciones más subjetivas de esas creencias, donde prima lo vivencial sobre lo doctrinario. Así, los hallazgos de esta fase de investigación 
indican una sintomatología propia de una "crisis latente de sentido" en palabras de Berger y Luckman (1997), que pone al descubierto la acuosidad de los sistemas de verdad religiosa, revelando problemas familiares en la transmisión de las creencias y las prácticas a las nuevas generaciones, transformaciones en la construcción de la identidad en su articulación con el discurso normativo religioso, así como distintas concepciones del papel de las Iglesias en su relación con el Estado y con la política. (BELGRAVES: 2013, p. 87)

Los datos obtenidos a partir de esta encuesta señalan retos significativos para la educación religiosa en los centros educativos públicos, ya que el escenario religioso que caracteriza la actualidad costarricense ya no es catolicocéntrico, eclesiocéntrico, ni tampoco exclusivamente teocéntrico. La actualidad tiene semblantes de espiritualidades diversas en un mundo plural. Un primer cuestionamiento se dirige al sistema político-educativo costarricense en el sentido de identificar su disposición para asumir el desafío de una educación religiosa acorde con el diálogo respetuoso y la convivialidad multirreligiosa. Además, el paso hacia cambios curriculares en este sentido conllevan a la necesidad de motivar y propiciar la formación de las personas docentes para un nuevo tipo de educación religiosa. Estos son algunos de los desafíos y necesidades que se vislumbran en el escenario de la educación religiosa en la Costa Rica actual.

Considerando la multiculturalidad y la multireligiosidad que caracterizan Costa Rica, ¿cómo desvelar y revelar, entre seguridades y dudas epistemológicas, una pedagogía caminante, que asume la ambivalencia, la provisoriedad y las pluralidades religiosas de la cotidianidad no como un problema, sino como una riqueza metodológica? ¿Cómo despedirse de los esencialismos y fundamentalismos pedagógicos y religiosos que pueden estar presentes en la educación religiosa? ¿Cómo construir aprendizajes más allá de los dogmatismos y sectarismos pedagógicos y religiosos?

Para abordar estas inquietudes pedagógicas y ensayar algunos cambios significativos para la educación religiosa, es necesario empezar con un acto de valentía: asumir una postura de transgresión educativa que posibilite el des-aprendizaje de las epistemologías pedagógicas universalizantes y homogeneizantes, para inventar una epistemología indócil, que rechaza el confort de las categorías conocidas. Es decir, se trata de idear una epistemología que genere pedagogías caminantes, que no absolutizan teorías educativas, sino que transitan por senderos no lineales, asumiendo una metodología relacional en una tensión permanente que no puede ser sintetizada en esquemas pedagógicos homogéneos. Las pedagogías caminantes son pedagogías en transgresión 
y gratuidad, que arman tiendas apenas para evaluar y recomenzar la marcha educativa después de un alto. Esto es lo que quiere provocar esta reflexión: hacer un alto en el camino para reflexionar sobre ciertas ideas educativas para, quizá, encontrar algunas comprensiones refrescadas y nuevas posturas docentes para la educación religiosa en Costa Rica y en América Latina.

\section{UNA EPISTEMOLOGÍA INDÓCIL QUE ANHELA EL RESPETO EN LAS DIVERSIDADES Y Y EL DIÁLOGO INTERRELIGIOSO}

Para entender el significado y el alcance de la expresión epistemología indócil en el marco de una perspectiva pedagógica nutriente de convivialidad y respeto en las diversidades, es importante mirar el escenario de la historia de la educación costarricense y latinoamericana para constatar la inexistencia, o por lo menos la insuficiencia, de prácticas y enfoques respetuosos con las diversidades que conforman los pueblos y las comunidades educativas. Además, es preciso advertir que, aunque ya se visualice un respetable acercamiento teórico a lo que se denomina educación inclusiva, las prácticas educativas conversacionales y promotoras de convivialidad todavía no se han incorporado satisfactoriamente en los escenarios latinoamericanos, invariablemente embarazados de muchas diversidades.

Por un lado, la escuela inclusiva es entendida como el centro educativo que recibe e incorpora a las personas estudiantes que presentan alguna discapacidad motora o cognitiva, o como el centro educativo que instaura de forma satisfactoria las adecuaciones curriculares, conforme sugiere la definición presentada por Peters:

La educación inclusiva puede entenderse como un principio rector destinado a alcanzar niveles razonables de integración escolar de todos los estudiantes. En el contexto de una visión más amplia de la integración, la educación inclusiva supone la formulación y aplicación de una vasta gama de estrategias de aprendizaje que respondan precisamente a la diversidad de los educandos. En este sentido, los sistemas educativos deben responder a las expectativas y necesidades de los niños y jóvenes teniendo en cuenta que la capacidad de ofrecer oportunidades reales de aprendizaje sobre la base de un esquema "rígido" de integración es muy limitada. Esto es lo que se puede calificar de paradigma de colocación. (PETERS: 2008, p. 10)

La incorporación de las personas estudiantes que presentan algún tipo deinhabilidad en los centros educativos ya es un importante paso, y es necesario reconocerlo. Sin embargo, esto no significa necesariamente la real 
2 Organización de las Naciones Unidas para la Educación, la Ciencia y la Cultura. inclusión de estas personas en los procesos de enseñanzaaprendizaje. Además, volvamos al cuestionamiento presentado anteriormente: ¿en qué sistema educativo se realiza la inclusión y cuáles son sus objetivos?

Por lo general, los sistemas educativos, incluyendo el sistema educativo costarricense, siguen promoviendo la competencia e insisten en reforzar la homogeneización del aprendizaje, especialmente de la educación religiosa, a través de contenidos, textos, procesos, tiempos y espacios, irrespetando las diversidades y menospreciando la necesidad urgente de aceptar, convivir y aprender con y en las diferencias.

Por otro lado, la definición de educación inclusiva que sugiere la $\mathrm{UNESCO}^{2}$ presenta la inclusión como un principio, es decir, la inclusión es considerada una cuestión crucial para que se logre una educación de calidad para todas las personas. La propuesta conceptual que ofrece la UNESCO reconoce las disparidades en los procesos de educación relacionadas con el género, las etnias, las condiciones socioeconómicas y las discapacidades físicas y cognitivas, y a partir de estas discrepancias ubica la perspectiva educativa inclusiva. Por ende la perspectiva conceptual presente en los documentos de la UNESCO presupone integración e inclusión:

La educación inclusiva puede interpretarse como un proceso continuo en un sistema educativo en permanente evolución que prioriza los que actualmente no pueden acceder a la educación y en los que están escolarizados pero no aprenden. No obstante, el concepto de la educación inclusiva no es frecuentemente bien comprendido y hay variadas interpretaciones del mismo en todo el mundo. (UNESCO: 2008, p. 9)

3 Los instrumentos normativos internacionales que han ratificado este derecho son, entre otros: la Convención relativa a la Lucha contra las Discriminaciones en la Esfera de la Enseñanza, de la UNESCO, 1960; el Pacto Internacional de Derechos Civiles y Políticos, de 1966; la Convención sobre la eliminación de todas las formas de discriminación contra la mujer, de 1979 (en especial los artículos 10 y 14); la Convención sobre los Derechos del Niño, de 1989 (especialmente los artículos 28 y 29); la Convención Internacional sobre la protección de los derechos de todos los trabajadores migratorios y de sus familiares, de 1990 (en particular el artículo 45); la Convención sobre los derechos de las personas con discapacidad, aprobada por la Asamblea General de las Naciones Unidas en diciembre de 2006 (especialmente el artículo 24); y la Declaración de las Naciones Unidas sobre los derechos de los pueblos indígenas, aprobada por la Asamblea General en septiembre de 2007 (particularmente el artículo 14).
La UNESCO ubica la educación inclusiva en el marco del derecho a la educación, consagrado por el artículo 26 de la Declaración Universal de Derechos Humanos de 1948. ${ }^{3}$ Parece estar claro que el locus de inclusión educativa defendida por la UNESCO es el sistema escolar, es decir, no se cuestionan las posibles deficiencias de éste sistema, ni tampoco se contempla una perspectiva más amplia de los procesos educativos que acontecen en otros espacios y momentos de la convivencia humana. Dice el documento:

La educación inclusiva puede considerarse como un proceso de fortalecimiento de la capacidad del sistema educativo para atender a todos los educandos. Por consiguiente, es un principio general que debería guiar todas las políticas y prácticas educativas, partiendo de la convicción de que la educación es un derecho humano fundamental y el fundamento de una sociedad más justa. Esta filosofía cimentada en los derechos se esboza en las declaraciones, convenciones e informes 
internacionales relevantes para la educación inclusiva. (UNESCO: 2008, p. 12)

La perspectiva pedagógica nutriente de convivialidad y respeto en las diversidades busca ir más allá de la inclusión educativa, al plantear procesos educativos innovadores y transgresores de los fundamentalismos epistemológicos y religiosos. El desafío epistemológico que se deriva de esta perspectiva es complejo y se configura como una tarea colectiva que exige coraje pedagógico, pues trata de generar una epistemología totalmente enredada - o entretejida con la práctica educativa y con la cotidianidad diversa de personas diversas, involucradas en procesos educativos diversos, en contextos diversos y con creencias diversas. En este sentido, la epistemología que se va generando no puede ser otra que una epistemología indócil, es decir, una epistemología que no se conforma con uniformidades y determinismos, tanto en el ámbito pedagógico (sistemas educativos) como en el religioso (sistemas eclesiásticos).

Lo que se busca, pues, es una mezcla revitalizadora de la práctica educativa cotidiana y el discurso sobre la misma, incluyendo los desafíos, diversidades y posibilidades del contexto, en un constante proceso de retroalimentación, cuestionamiento, revisión, desaprendizajes, aprendizajes, desinstalación, re-creación, disfrute, resistencia y reconstrucción. Desde esta perspectiva, la educación religiosa en los centros educativos asume importancia y relevancia.

En la misma dirección, el brasileño João Décio Passos ubica la educación religiosa como una disciplina necesaria en los actuales currículos escolares, e introduce la reflexión sobre la necesidad de una fundamentación epistemológica para la educación religiosa. Dice el autor:

El estudio de la religión rompe con la epistemología moderna centrada en la práctica monodisciplinar $\mathrm{y}$, por ende, en la visión fragmentada de la realidad; epistemología que, por lo menos en sus abordajes hegemónicos, negó lugar a la religión y, muchas veces, a su estudio. La enseñanza religiosa asume, necesariamente, en sus definiciones curriculares, la crítica al conocimiento en el dominio de algún aspecto de la realidad, la crítica al positivismo que entiende la ciencia como única versión de la verdad y la crítica a la neutralidad de las ciencias como abordaje definitivo de la realidad. El estudio de la religión incluye, en sí mismo, la confrontación con la pluralidad de modelos, la decodificación de experiencias valorativas que involucran a los sujetos, el abordaje de cuestiones que trascienden pragmatismos teóricos y sociales y la búsqueda de valores que fundamentan la convivencia humana. (PASSOS: 2007, p. 43) 
La perspectiva epistemológica presentada por Passos coincide con el enfoque epistemológico del cual estamos hablando, es decir, una epistemología en movimiento, en creación, inacabada, que sugiere, además, que la reflexión y producción académicas deben expresar el involucramiento personal, humano y profesional de las personas educadoras. Esto significa que es necesario vincular las reflexiones con las experiencias, los desaprendizajes con los aprendizajes, buscando romper con la verticalidad y la supuesta imparcialidad que caracterizan la mayoría de las relaciones educativas y también la producción académica.

Se entiende, pues, que la perspectiva pedagógica que nace de la indocilidad epistemológica posibilita ensayar nuevos compromisos en la docencia, especialmente en la docencia relacionada con la educación religiosa. Esto porque representa la posibilidad de una ruptura de los ciclos de uniformización y exclusión educativas, y ofrece una formación diferenciada a las personas estudiantes, desde la convivialidad y el respeto en las diversidades.

\section{HACIA UNA PERSPECTIVA METODOLÓGICA RELACIONAL: LA CONVERSACIÓN INTERCULTURAL QUE NUTRE LA CONVIVIALIDAD}

En el marco de una epistemología indócil, la perspectiva pedagógica nutriente de convivialidad y respeto en las diversidades asume una opción metodológica que denominamos relacional, porque tiene que ver con metodologías que promueven conversaciones y alimentan relaciones saludables. En este sentido, la relacionalidad metodológica no debería incidir solamente en los espacios convencionales del quehacer educativo, sino también en la construcción de puentes entre la docencia y el contexto sociocultural, entre las creencias y no-creencias, entre las diversas expresiones y memorias culturales, entendidas no como categorías reunidas, sino como áreas intrínsecamente inter-relacionadas. En otras palabras, se trata de metodologías en libertad y en movimiento que se arriesgan a ir más allá de los altos muros de algunos espacios epistemológicos para pensar, decir y conocer desde otras espacialidades y otras temporalidades. Son metodologías que siguen las interpelaciones de la cotidianidad, son provisorias, sin que esto signifique que sean indefinidas o que se les falte una fundamentación; son metodologías que beben las palabras, las vivencias y los dolores de la actualidad y no pueden ser aplicadas de forma equivalente para todos los tiempos y lugares.

En ese sentido, las metodologías embarazadas de la perspectiva metodológica relacional necesariamente interpelan los presupuestos investigativos clásicos, es decir, las formas de pensar y de investigar que se apoyan en el dualismo entre la persona investigadora que conoce y el objeto por conocer. La relacionalidad metodológica 
4 Sobre la otredad y los otros rostros, véase las siguientes obras de Emmanuel Levinás: Entre nosotros. Ensayos para pensar en otros. Valencia: Pre-Textos, 1993; y Nombres propios. Madrid: Fundación Emmanuel Mounier, 2008. rompe no solamente con este dualismo, sino también con el individualismo cognitivista, antropocéntrico y androcéntrico que, como sugiere Fornet-Betancourt, imposibilitan la experiencia del conocimiento como experiencia de participación y convivencia. (BERISSO; GIULIANO: 2015, p. 157)

Por otro lado, las metodologías relacionales sugieren que los cambios no pueden acontecer solamente en la esfera de las ideas, sino también en las relaciones mismas de la vida, lo que significa vislumbrar un quehacer pedagógico interpelado por el rostro y la historia de las otredades. ${ }^{4}$ En este sentido, Fornet-Betancourt presenta una interesante reflexión sobre la interculturalidad y la necesidad de recordar que las memorias que somos tienen que ver con las memorias de la otredad y, por ende, el camino hacia el conocimiento pasa por los rostros y las interpelaciones de las demás personas, es decir, el propio conocimiento se convierte en un camino relacional:

Comprender al otro, acogerlo en su otredad y hospedarlo, es siempre un proceso queimplica escuchar el reclamo de vida y mundo propios que representa la alteridad del otro; por eso no se puede quedar en una experiencia abstracta de abertura cognitiva sino que se tiene que concretar en la repartición justa de la realidad histórica, en una política de restitución que complementa el reconocimiento con la devolución de lo que se le ha negado. En esta doble y complementaria dialéctica se mueve la propuesta de la filosofía intercultural. (BERISSO \& GIULIANO: 2015, p. 157)

Entendemos que la efectiva implementación de metodologías relacionales en los procesos de educación religiosa significa una posibilidad muy concreta de generación de espacios-tiempos educativos desde otros marcos metodológicos para mirar hacia las otredades religiosas y las diversidades religiosas, para recibirlas y hospedarlas. Los procedimientos educativos que se derivan de las metodologías relacionales son también promotores de relacionalidades, es decir, generadores de una educación de cercanía, de alegrías y sufrimientos compartidos, de sorpresas como materia de reflexión. Por esto, al hablar de otredades religiosas, o de relación y relacionalidades inter-religiones, la educación religiosa queda ubicada en la cotidianidad, lo que significa reconocer los saberes y sabidurías de la gente, sus creencias y no-creencias, su fe y sus temores, y asumir una ética del cuidado inspirada en las historias religiosas personales y colectivas.

Las metodologías relacionales hacen que la educación se convierta en un espacio-tiempo de decir el mundo y de búsqueda de caminos y respuestas para la eterna sed humana de aprendencia y de espiritualidad. Esto porque las relacionalidades cargan en sí mismas la oportunidad de encuentro con las otredades y el encuentro con los propios 
recuerdos, memorias, aprendizajes y fe religiosa; tal vez también provoquen el encuentro con miedos y cicatrices, sonrisas y nostalgias, culpas y reconciliaciones, y esto se convertirá en insumo importante para la educación religiosa.

Otro aspecto muy significativo en la propuesta metodológica relacional es la concerniente a la temporalidad en/de los procesos educativos, ya que las metodologías relacionales implican que la temporalidad dominante, lineal y orientada al progreso, no coincide con las temporalidades plurales construidas desde las diversidades y la interculturalidad. Méndez cuestiona la organización del tiempo educativo de la siguiente forma:

Los procesos educativos socializan y transmiten una determinada forma de organizar el tiempo; generan experiencias de tiempo: horarios, calendarios, efemérides, rituales, ciclos trimestrales, bimestrales, semestrales, anuales, etc. La educación - como las demás actividades humanas- acontece en el tiempo y produce una temporalidad, es decir, produce conciencia de tiempo y enseña a tener control sobre el tiempo. Esa conciencia de tiempo no se produce solo desde el discurso y los contenidos. Se produce sobre todo desde la metodología, la evaluación y todo lo que constituye eso que ha sido llamado currículo oculto. (...) Quiero sugerir aquí la posibilidad -y la necesidadde generar procesos educativos en los que, superando la colonización "totalitarista utilitarista y consumista del tiempo, pensemos y actuemos temporalidades plurales cargadas de memorias y esperanzas. (MÉNDEZ: 2010, p. 147)

El mismo autor se refiere a la educación y sus ambivalencias con confianza, lo que abre escenarios de esperanza educativa también para la educación religiosa mediatizada por las relacionalidades:

La educación es siempre una mediación: puede serlo para la conservación o para la transformación, para el consenso o para el disenso, para la imposición o para la proposición, para la violencia o para la reconciliación, para el sometimiento o para la resistencia y la emancipación. Por eso, la educación es una mediación ambivalente: depende de lo que se quiera hacer con ella, o mejor, depende del ¿̇para qué? (MÉNDEZ: 2008, p. 103)

De acuerdo con las reflexiones anteriores sustentadas por el pensamiento de Fornet-Betancourt y Méndez, queda claro que la apertura a las otredades diversas y el respeto intercultural se constituyen como el alimento metodológico 
primordial para las prácticas educativas nutrientes de convivialidad y respeto en las diversidades. A través de la vivencia efectiva de la interculturalidad, es posible la generación de convivencias armoniosas que nacen de las controversias y las diferencias. Fornet-Betancourt, al referirse al diálogo intercultural, dice lo siguiente:

Ciertamente quiere la con-vivencia la armonía, sin embargo esta no debe nacer por la vía rápida de la apropiación reduccionista, como ha intentado tantas veces la racionalidad occidental. La con-vivencia, al contrario, marca la armonía que se iría logrando por la constante interacción en el campo históricopráctico y por la subsiguiente plataforma intercomunicativa que irían tejiendo los discursos en la misma explicación de sus contro-versias. La con-vivencia apunta así a esa forma superior de armonía que puede designarse con el nombre de solidaridad. La solidaridad supone y quiere al otro desde su alteridad y exterioridad. (FORNETBETANCOURT: 1994, p. 23)

Por fin, como un intento de nutrir la esperanza educativa en la educación religiosa, resaltamos que una de las posibilidades de las metodologías relacionales tiene que ver con el hecho de ser la educación un lugar de encuentro. Eso es así simplemente porque la educación acontece en espacios y tiempos de vida que están inacabados, en dónde las personas necesitan encontrarse, relacionarse, con-vivir, y cuyas dimensiones y potencialidades escapan a cualquier intento de control o conocimiento definitivo. Hacer de la educación un lugar de encuentro, algo como un taller para hacerse persona pude ser pretencioso, pero este es el principal desafío que presentan las metodologías relacionales para la educación religiosa. Más que una disciplina con sus cánones - epistemológicos, metodológicos y religiosos -, la educación religiosa puede ser una de las mediaciones hacia la convivialidad, y es tarea de las personas educadoras comprender esa posibilidad para transformarla en práctica educativa.

\section{DESAFíos ACTUALES PARA LA EDUCACIÓN RELIGIOSA EN COSTA RICA: EL ATERRIZAJE DE LA EPISTEMOLOGÍA INDÓCIL Y LAS METODOLOGÍAS RELACIONALES EN LAS PRÁCTICAS EDUCATIVAS}

Elcultivodeunaeducaciónquerespetelasdiversidades y promueva la convivialidad es un aprendizaje que debe iniciarse en la más temprana edad y debe ser impulsado desde los centros educativos. Así, en el marco de una epistemología indócil y desde la perspectiva metodológica relacional, ¿̇cuál es el aporte que puede ofrecer la educación religiosa para la sociedad costarricense? 
Educar tiene consecuencias e implicaciones, ya que la educación representa un espacio social complejo, portador de posibilidades y también de contradicciones. La educación religiosa en Costa Rica vive actualmente un periodo de muchas posibilidades, como por ejemplo la construcción de una educación de carácter laico y el desarrollo de estudios críticos de las religiones. El énfasis tradicional de la educación religiosa costarricense ha sido la transmisión de la doctrina y la moral católica, con indicadores eclesiásticos para medir el grado de aprendizaje de las personas estudiantes. El giro necesario pasa por las relacionalidades, es decir, la dimensión horizontal subyacente que expresa el desarrollo espiritual humano y produce un impacto relacional positivo.

En el aterrizaje de la perspectiva epistemológica indócil y de las metodologías relacionales, es importante no idealizar la convivialidad o abordarla de manera superficial a través de expresiones como mundo armónico, relaciones armoniosas, sino asumir que las implicaciones éticas radicales de la convivialidad pasan también por la educación religiosa. ¿Cómo traducir esto en la dinámica de las clases de educación religiosa? El punto de partida es, sin duda, el reconocimiento de la multirreligiosidad existente en Costa Rica para hacer síntesis y sincretismos y suplantar la catequesis católica de los espacios de educación pública.

Sin la pretensión de ofrecer respuestas generalizadas o recetas educativas, indicamos algunas posturas que pueden ser asumidas por las maestras y maestros de religión e instituciones educativas, frente al desafío de reconstruir la educación religiosa:

- Prepararse responsablemente para una nueva etapa de la educación religiosa en el país desde el marco de la interculturalidad y la multirreligiosidad.

- Comprender las raíces coloniales del actual modelo de educación religiosa.

- Ver la exclusión religiosa en los procesos educativos en dependencia con otras categorías de opresión y desigualdad.

- Cultivar la criticidad y la lucidez: percibirse dependientes de verdades condicionadas y promulgadas por las instituciones educativas $\mathrm{y}$ religiosas.

- Entender que los debates sobre educación religiosa van más allá de las cuestiones de la institucionalidad educativa.

- Rechazar los contenidos religiosos laureados y legitimados por el poder eclesiástico.

- Cuestionar dogmatismos, fundamentalismos e institucionalidades violentas.

- Rechazar el elitismo científico y la retórica religiosa.

- Impulsar una educación religiosa que desorganiza esquemas y planes y que no se satisface con el confort de las clases, de los auditorios e iglesias. 
- Salir de la abstracción y de los esquemas conceptuales impuestos, que buscan ofrecer respuestas sencillas y absolutas para problemas complejos y cambiantes.

- Recuperar los conocimientos y las espiritualidades experimentadas por personas que fueron ocultadas de los procesos de contar y hacer la historia de la educación, y también la historia de las espiritualidades.

- Cultivar relaciones armoniosas, desde las relacionalidades interpersonales hasta las relacionalidades con el medioambiente y el cosmos.

- Proponer una educación religiosa que incentive la fiesta, la alegría y la risa, una educación religiosa juguetona, con características esféricas, para metaforizar una nueva manera de ser en relación, en igualdad y en circularidad.

Estos son apenas algunos de los desafíos didácticos que se presentan en una sociedad religiosamente diversa. Sin duda, la educación religiosa mediada por la perspectiva epistemológica indócil y por las metodologías relacionales supone una buena dosis de altruismo, que es la calidad que hace que los seres humanos cooperen y sean solidarios. Por otro lado, es importante elucidar que la tarea de reinventar la educación religiosa no es una labor solitaria de las maestras y maestros, sino una responsabilidad de todas las personas que se preocupan por la educación de las niñas, niños y adolescentes en los centros educativos públicos de Costa Rica.

El temor de muchas personas educadoras ha sido cómo reinventar la educación religiosa en las aulas, y esta es una inquietud muy pertinente, ya que nuevos marcos pueden provocar vacíos y ambigüedades. Sin embargo, la alternativa de una educación religiosa generadora de convivialidad y respeto en las diversidades no significa tener un documento único o una receta pedagógica, pues cada persona educadora y cada centro educativo deberían indagar sobre lo religioso a partir de los contextos locales y las necesidades que de ellos se derivan. Por esto, en la tarea de ir re-creando la educación religiosa, es muy probable que se logre ver realidades nuevas y diversas que habitan los espacios educativos. Tal vez ni sean tan nuevas, pero podrán ser vistas y descubiertas desde otra mirada.

\section{REFERENCIAS BIBLIOGRÁFICAS}

BELGRAVES, L. F. ¿Un menú de creencias a fuego lento?: Acercamiento sociológico a la religión en Costa Rica. En: Revista Siwo, Volumen 7, Número 1. San José: Sebila, 2013.

BERISSO, D.; GIULIANO, F. La educación como práctica de convivialidad. Una conversación con Raúl FornetBetancourt. En: Revista del Cisen Tramas / Maepova. 
Vol. 3, $\mathrm{N}^{\circ}$ 1. Abril de 2015. Versión digital disponible en http://ppct.caicyt.gov.ar/index.php/cisen/article/ view/6917/6237

FORNET- BETANCOURT, R. De la convivencia a la convivialidad. Una introducción. En: Denktraditionen im Dialog - Studien zur Befreiung und Interkulturalität, Band 32. Alemania: Verlag Mainz. 2011.

FORNET- BETANCOURT, R. Hacia una filosofía intercultural latinoamericana. San José: DEI, 1994.

FREIRE, P. Extensão ou comunicação? $10^{\mathrm{a}}$ Ed. Rio de Janeiro: Paz e Terra, 1983.

LEVINÁS, E. Nombres propios. Madrid: Fundación Emmanuel Mounier, 2008.

Entre nosotros. Ensayos para pensar en otros. Valencia: Pre-Textos, 1993.

MÉNDEZ, J. M. Temporalidad y educación: la construcción de temporalidades humanizantes desde los procesos educativos. En: Revista de Teología Siwo, $\mathrm{N}^{\circ} 3$, 2010. San José: Sebila.

MÉNDEZ, J. M. La mediación educativa frente al cultivo de la violencia: un aporte desde la perspectiva intercultural. En: Revista de Teología Siwo, No 1, Volumen 1, 2008.

PASSOS, J. D. Ensino religioso: construção de uma proposta. Coleção Temas do Ensino Religioso. São Paulo: Paulinas, 2007.

PETERS, S. Inclusive education: An EFA strategy for all children, Washington DC, Banco Mundial, 2004. En: UNESCO, Conferencia Internacional de Educación, 48 reunión, La educación inclusiva: el camino hacia el futuro. UNESCO, Ginebra: 2008.

MATURANA, H. Transformación en la convivencia. Chile: Comunicaciones Noreste, 2004.

UNESCO, Conferencia Internacional de Educación, 48 reunión, La educación inclusiva: el camino hacia el futuro. UNESCO, Ginebra: 2008.

Recebido em: 11/05/2018

Aprovado em: 07/08/2018

Publicado em: 31/o8/2018 